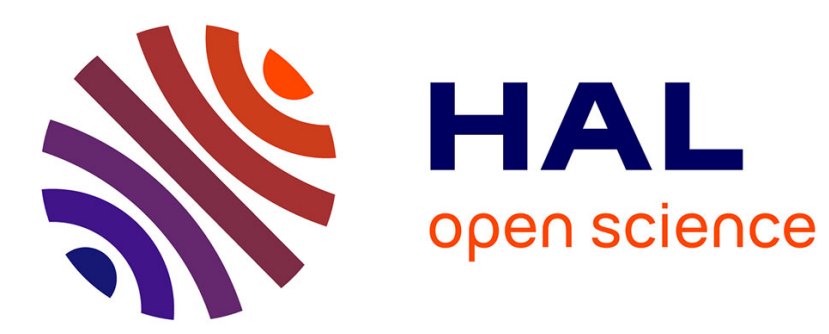

\title{
Numerical and experimental investigation of nonlinear properties of rubber absorber in rail fastening
}

\author{
Yanyun Luo, Yan Liu, Yin Hai-Ping
}

\section{To cite this version:}

Yanyun Luo, Yan Liu, Yin Hai-Ping. Numerical and experimental investigation of nonlinear properties of rubber absorber in rail fastening. International Journal of Mechanical Sciences, 2013, 69, pp.107113. 10.1016/j.ijmecsci.2013.01.034 . hal-00850858

\section{HAL Id: hal-00850858 https://hal.science/hal-00850858}

Submitted on 12 Aug 2013

HAL is a multi-disciplinary open access archive for the deposit and dissemination of scientific research documents, whether they are published or not. The documents may come from teaching and research institutions in France or abroad, or from public or private research centers.
L'archive ouverte pluridisciplinaire HAL, est destinée au dépôt et à la diffusion de documents scientifiques de niveau recherche, publiés ou non, émanant des établissements d'enseignement et de recherche français ou étrangers, des laboratoires publics ou privés. 


\title{
Numerical and experimental investigation of nonlinear properties of rubber absorber in rail fastening
}

\author{
LUO Yanyun, LIU Yan, Yin H.P.
}

Building F212, Jiading Campus, Tongji University, 4800\# Caoan Road, Shanghai, 201804

\begin{abstract}
A dynamic nonlinear model of rubber absorber in railway fastening is proposed in this paper based on a superposition principal demonstrating that the restoring force of rubber components consists of an elastic force and a damping force. In order to determine model parameters and verify the accuracy of this model, a dynamic experiment is designed. The obtained simulated results show good agreement with the measured counterparts, which means that the proposed dynamic nonlinear model should therefore be an effective mechanical tool in simulation and characterization of dynamic nonlinear behavior of rubber absorber in rail fastening with certain modes of vibrations. Excitation frequency dependency and amplitude dependency of the dynamic nonlinear stiffness were also studied. The result indicates that characteristics of the dynamic stiffness is closely associated with both displacement amplitude and frequency, the latter however is not as great as the former.
\end{abstract}

Keywords: Rubber absorber, Rail fastening, Dynamic stiffness, Parameter identification

\section{Introduction}

Rubber material has found an indisputable role in many mechanical applications due to its high elongation, incompressibility, reversibility, high mechanical strength, long-term stability of their hardness and service life. Specifically, it could withstand deformation up to several hundred percents while resuming its original shape after a certain time since stress release. This perfect elastic property facilitates its wide use as a flexible coupling between stiff components in various constructions, e.g. vibration isolators, suspensions and flexible joints.

Although engineering knowledge of rubber material is still rather poor probably due to its complex nonlinear mechanical properties, the common use of rubber material has made it a subject drawing prior attention all over the world. It has been generally believed that dynamic stiffness and damping are dependent not only on additives in the material but also on temperature, geometry, frequency and amplitude of motion [1-5]. Additionally, thorough studies on the two 
well-known stress-softening effects ---Mullins effect and Payne effect---have been given long time ago [4-7]. Subjects focusing on the influence of fillers on dynamic properties have been discussed as well [8-11]. There are many other works investigating relatively complex measurement conditions of rubber components [12-15]. Besides these theoretical knowledge mentioned above, studies about applications of rubber material such as isolators of electronic equipments, machines, building constructions and engine mounts reducing transmission of engine vibrations to the chassis of vehicles driven over rough road surfaces have been proposed [16-18]. However, specialized researches focusing on the dynamic properties of rubber absorber in rail fastening is rarely found up to now, even though it has been widely used in fastening systems.

Rail fastening plays an important role in the whole track structure as a vibration reduction system, whose elasticity determined mainly by its rubber components is directly related to train operation property and comfort. The key requirement for high quality of elastic rail fastening is to optimize the dynamic parameters during designing process and consequently to facilitate its effective use as a vibrating reduction device in the whole wheel-rail system [19-20]. Thus, understanding the nonlinear characteristics of rubber absorber in rail fastening as well as optimizing dynamic stiffness and damping is increasingly important, especially since the high-speed railway claims much higher demands on elasticity of rail fastening because of its relatively large-scale motion.

It is worth noting that rubber absorber in rail fastening, not like most rubber damping devices in other applications, is subjected to a static pressure force all the time due to the superstructure force such as train, rail and clamping force of fasteners apart from various dynamic forces. Therefore, numerical and experimental investigations of dynamic nonlinear properties of rubber absorber in rail fastening have been done in this paper. An initial displacement resulted from a pre-pressure and additional displacement excitations are both included in the measurement condition to simulate the real working condition of rubber absorber in rail fastening during the dynamic experiment. In order to clearly show the differences between common rubber devices used in most applications for alleviating the severity of vibration problems and rubber absorber in rail fastening, Fig.1 shows the well-known hysteresis loops of rubber component without pre-pressure [21] and in rail fastening with certain pre-pressure $F_{0}$ respectively, where $x_{0}$ is correspondingly the initial displacement. 


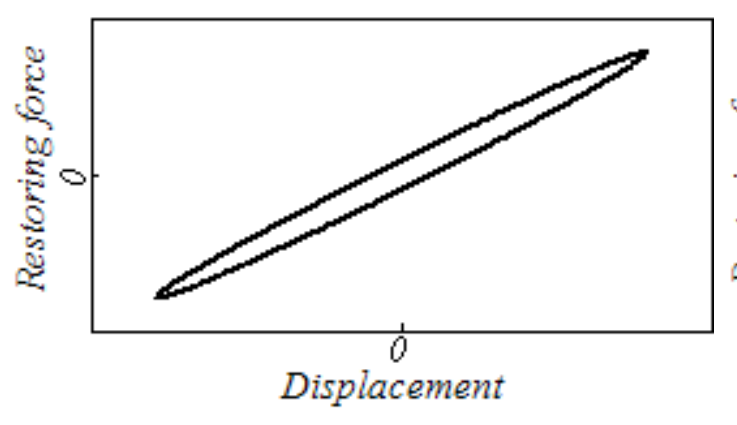

Fig. 1-1 Force-displacement loop of rubber absorber without pre-pressure

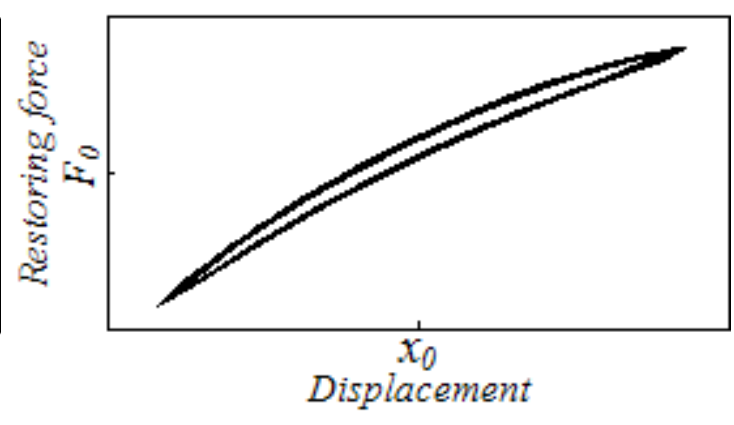

Fig. 1-2 Force-displacement loop of rubber absorber in rail fastening

Fig.1 Comparison diagrams of force-displacement loop between rubber absorber without pre-pressure and that in rail fastening with pre-pressure

A nonlinear dynamic model consisting of two parts - the nonlinear elastic force and the nonlinear damping force- - based on the viscoelastic mechanical theory is proposed to simulate the dynamic nonlinear behavior of rubber absorber in rail fastening when it is exposed to harmonic excitations.

The model mentioned above is not only used to reveal the strong nonlinear properties as rubber absorber in rail fastening works in its special working environment mentioned above, but is also concerned with the amplitude and frequency dependency of dynamic stiffness. After a procedure to indentify all the model parameters, the accuracy of this model is verified by comparing the measured and simulated results.

\section{Modeling}

Although several attempts to describe the vibration behavior of rubber material have been achieved, most proposed models are only applicable to certain applications primarily due to their given working conditions. It has been generally believed that the restoring force of rubber components subjected to harmonic excitations consists of two parts: the damping force and the elastic force. That is to say the total restoring force can be obtained from the direct summation of the two parts as

$$
F_{\text {Restoring }}=F_{c}+F_{k}
$$

where the former term describes damping force with energy loss, and the latter is incorporated to describe elastic force without any energy loss. Throughout this work the study is based on this superposition principle. The force-displacement hysteresis loop of rubber absorber in rail fastening 
shown in Fig.1-2 could be decomposed into two parts accordingly, and the result is displayed in Fig.2, where $A$ is displacement amplitude.

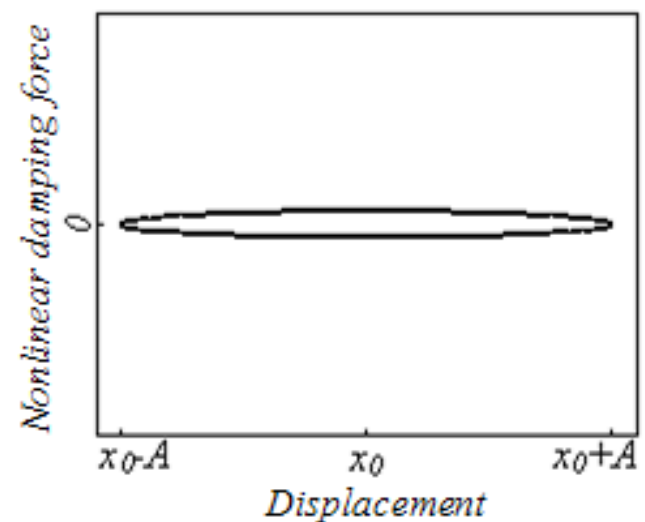

Fig. 2-1 Nonlinear

damping force-displacement curve

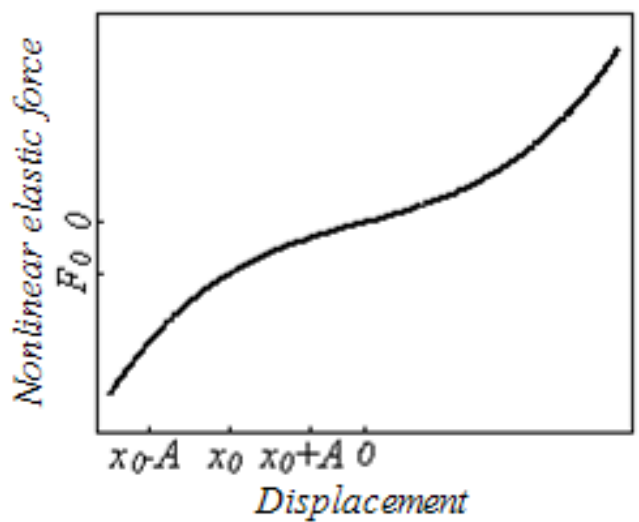

Fig. 2-2 Nonlinear elastic force-displacement curve

Fig.2 Decomposition diagram of force-displacement loop of rubber absorber in rail fastening

In order to simulate the nonlinear behavior of rubber absorber in rail fastening, it can be seen from Fig.2 that both damping force-displacement loop and the elastic force-displacement curve are supposed to be nonlinear curves. It should be noted that there is an initial displacement $x_{0}$ in Fig.2-1, i.e. the equilibrium position of $\mathrm{x}$-coordinate is not zero, and that the area of this closed curve is equal to that of the hysteresis loop in Fig.1-2, both of whom represent the energy loss of rubber absorber during working hours. Aiming at capturing the frequency-dependent and amplitude-dependent characteristics, the nonlinear elastic force and damping force are regarded as not constant but vary as functions of displacement frequency and amplitude. The influences of changed temperature and preload are supposed to be associated to a lesser degree with the dynamic nonlinear characteristics of rubber absorber in rail fastening, which are ignored in this paper.

\subsection{Nonlinear damping force}

According to the knowledge of nonlinear theory and the shape of force-displacement loop of rubber absorber in rail fastening, an ellipse is proposed to simulate the nonlinear damping force as the function of displacement, frequency and amplitude with an initial displacement $x_{0}$, and $\left(x_{0}, 0\right)$ is just the center point of the ellipse. The elliptic expression could be described as

$$
\frac{\left(x-x_{0}\right)^{2}}{A^{2}}+\frac{F_{c}(x, \omega, A)^{2}}{b^{2}(\omega, A)}=1 \text {, }
$$

where, $x, x_{0}, A, b$ are displacement, initial displacement, amplitude and an undetermined 
coefficient respectively, whose units are all $\mathrm{mm}$, and $F_{c}$ is the nonlinear damping force, $K N$. Since the area of the ellipse representing energy loss $E$ of rubber absorber during loading cycles is closely related to frequency and displacement amplitude [22], the undetermined coefficient $b$ could be obtained as followed according to elliptical area formula

$$
b(\omega, A)=\frac{E(\omega, A)}{\pi A}
$$

Merging equation (3) into (2), the nonlinear damping force of rubber absorber in rail fastening finally is

$$
F_{c}(x, \omega, A)= \pm b(\omega, A) \sqrt{1-\frac{\left(x-x_{0}\right)^{2}}{A^{2}}}= \pm \frac{E(\omega, A)}{\pi A} \sqrt{1-\frac{\left(x-x_{0}\right)^{2}}{A^{2}}}
$$

\subsection{Nonlinear elastic force}

As for the nonlinear elastic force-displacement curve, it is assumed to be a polynomial of power functions in the model, whose terms shall be determined according to the requirement of accuracy. This nonlinear elastic force is not only a function of displacement $x$, but also frequency $\omega$ and amplitude $A$ as the nonlinear damping force, so it could be thus described as

$$
F_{k}(x, \omega, A)=\sum_{i=1}^{(n+1) / 2} K_{2 i-1}(\omega, A) \cdot x^{2 i-1}=K_{1}(\omega, A) x+K_{3}(\omega, A) x^{3}+K_{5}(\omega, A) x^{5}+\cdots
$$

where the polynomial coefficients of each order are defined as dynamic stiffness $K_{i}(i=1,2 \ldots)$.

\subsection{Nonlinear restoring force}

The restoring force of rubber absorber in rail fastening exposed to harmonic excitations is the direct summation of nonlinear damping force and nonlinear elastic force based on the superposition principle mentioned above as

$$
F_{\text {Restoring }}(x, \omega, A)=F_{k}+F_{c}=\sum_{i=1}^{(n+1) / 2} K_{2 i-1}(\omega, A) \cdot x^{2 i-1} \pm \frac{E(\omega, A)}{\pi A} \sqrt{1-\frac{\left(x-x_{0}\right)^{2}}{A^{2}}}
$$

\section{Experimental}

In order to obtain model parameters, a dynamic experiment is designed. The measurements are also used to verify the accuracy of proposed model through comparing it to the simulated results. 


\subsection{Test object}

The test object is a real rail fastening system commonly used in China, see Fig.3. The dimension of the rubber component in it is $320 \times 180 \times 20 \mathrm{~mm}$. It is made up of mainly natural rubber and the rubber hardness is $65 \mathrm{Hs}$.

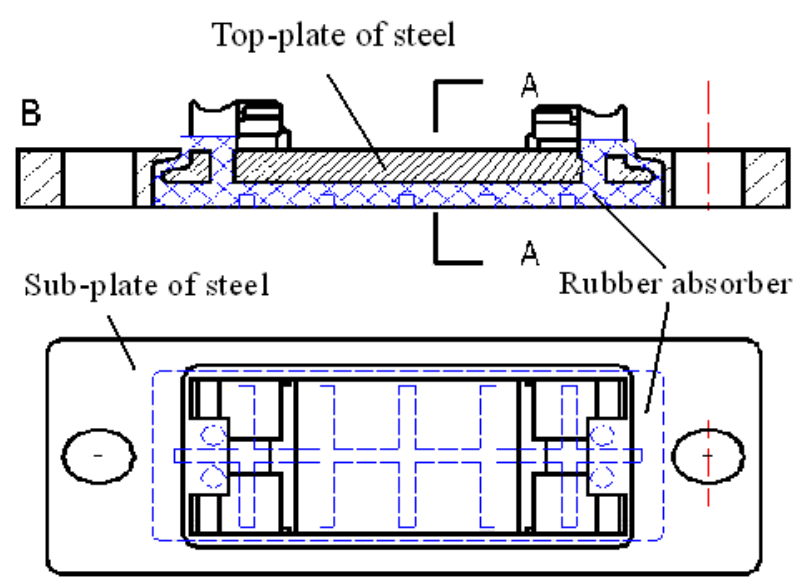

Fig.3 Shap of the test rail fastening

\subsection{Excitation signals}

Although rubber components in various vibration constructions are rarely exposed to dynamic single-tone force, it is the major measurement condition in most references, and additional softening effects are likely to occur when rubber components are subjected to more complex excitation signals [13]. Therefore, different single-tone sinusoidal displacement excitations are adopted during the measurements of this investigation, i.e. there is no superimposition of excitation signals. The sinusoidal excitation ranges in frequency from 2 to 10 $\mathrm{Hz}$ and in amplitude from 0.3 to $0.7 \mathrm{~mm}$, because rubber absorber in rail fastening bears a dynamic load with main frequency below $10 \mathrm{~Hz}$ and has a small displacement response. The excitation signals could be expressed as $x(t)=A \sin (\omega t)+x_{0}$, where $\omega$ is excitation frequency, $A$ is displacement amplitude and $x_{0}$ is the real initial displacement controlled by the minimum displacement, being recorded as mean position during the course of expeciment. The detailed excitation cases are shown in Table 1.

\subsection{Test description}

Measurement of force-displacement relationship of rubber absorber in rail fastening was performed by an Instron servohydraulic testing machine. The displacement excitation waves were applied through a hydraulic actuator, which was monitored by a variable differential transformer. 


\section{Table 1}

Excitation cases

\begin{tabular}{|c|c|c|c|c|c|}
\hline Number & $x_{0}$ & \multicolumn{4}{|c|}{ Case } \\
\hline \multirow[t]{5}{*}{$1-5$} & -0.900 & & & & $2 \mathrm{~Hz}$ \\
\hline & -0.900 & & & & $4 H z$ \\
\hline & -0.899 & Amplitude & $0.3 \mathrm{~mm}$ & Frequency & $6 H z$ \\
\hline & -0.903 & & & & $8 \mathrm{~Hz}$ \\
\hline & -0.900 & & & & $10 H z$ \\
\hline \multirow[t]{5}{*}{$6-10$} & -1.101 & & & & $2 \mathrm{~Hz}$ \\
\hline & -1.099 & & & & $4 \mathrm{~Hz}$ \\
\hline & -1.099 & Amplitude & $0.5 \mathrm{~mm}$ & Frequency & $6 \mathrm{~Hz}$ \\
\hline & -1.104 & & & & $8 H z$ \\
\hline & -1.104 & & & & $10 \mathrm{~Hz}$ \\
\hline \multirow[t]{5}{*}{$11-15$} & -1.300 & & & & $2 \mathrm{~Hz}$ \\
\hline & -1.291 & & & & $4 H z$ \\
\hline & -1.298 & Amplitude & $0.7 \mathrm{~mm}$ & Frequency & $6 \mathrm{~Hz}$ \\
\hline & -1.300 & & & & $8 H z$ \\
\hline & -1.305 & & & & $10 \mathrm{~Hz}$ \\
\hline
\end{tabular}

The restoring force is measured using a force transducer connecting with the top the test object, whereas the displacement is measured and controlled using two displacement transducers connecting with the bottom of the test object. In addition, the test system mainly consists of electrodynamic vibration shaker table, vibration control system, data acquisition system, data processing system, computer, and charge amplifier as well as power amplifier, see Fig.4

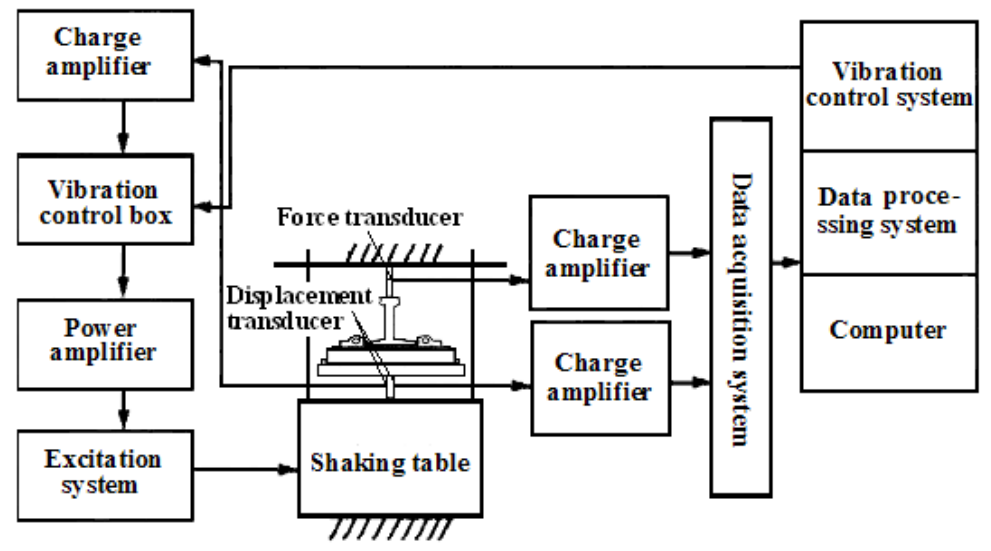

Fig.4 Diagram of test process 


\section{Determination of model parameters}

\subsection{Evaluation of dynamic stiffness}

By using the measured results, model parameters are evaluated through the least square method in this section. Coefficients of the polynomial of power function, being the dynamic stiffness of each order, are identified according to equation (6), while the coefficient $E$ of all excitation cases representing energy loss could be obtained based on MATLAB platform. The identified results of polynomial coefficients, as the number of polynomial terms $n$ values 3 and 5, are shown respectively in Fig.5-1 and Fig.5-2, where the excitation case as displacement amplitude is $0.3 \mathrm{~mm}$ and frequency is $2 \mathrm{~Hz}$ is taken as example. It is clear that the value of the fifth order dynamic stiffness, i.e. the value of parameter $P_{3}$ in Fig.5-2, is much fewer compared with the first and the third order dynamic stiffness, i.e. correspondingly the value of parameters $P_{l}$ and $P_{2}$. It is also evident that the identified results shown in Fig.5-1 where there are only the first and
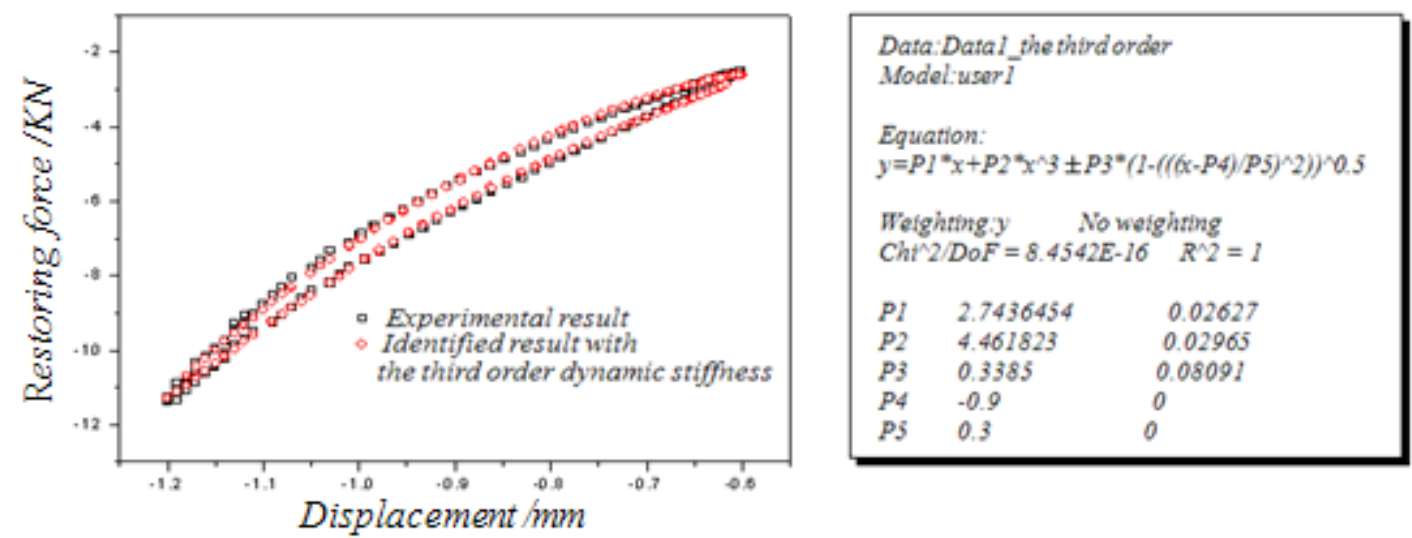

Fig.5-1 Identified results as the number of polynomial terms in equation (6) values 3
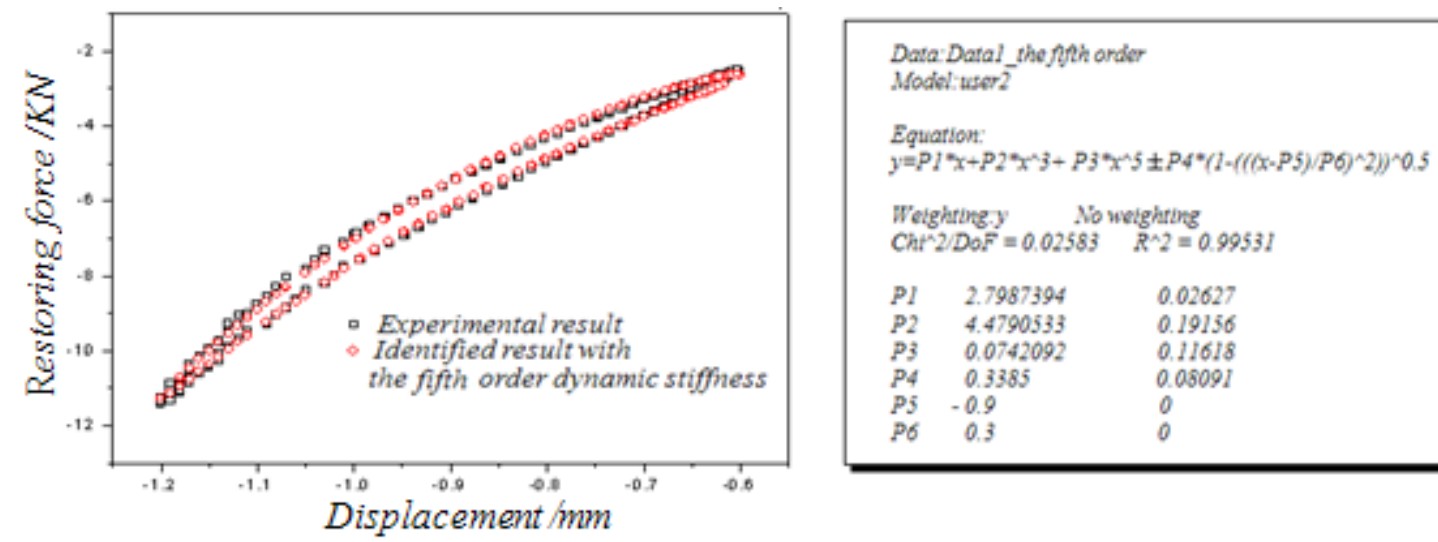

Fig.5-2 Identified results as the number of polynomial terms in equation (6) values 5

Fig.5 Identify results of the excitation case as displacement amplitude is $0.3 \mathrm{~mm}$ and frequency is $2 \mathrm{~Hz}$. 
the third order dynamic stiffness could already meet the accuracy demand by contrast with Fig.5-2 with a fifth order dynamic stiffness. Therefore, a dynamic model with only the first and the third order dynamic stiffness is studied in the following sections of this paper.Through the same method based on ORIGIN platform, a set of model parameters of all excitation case is obtained as shown in Table 2.

\section{Table 2}

Identified dynamic stiffness of each order for every excitation case

Frequency Amplitude The first order dynamic stiffness The third order dynamic stiffness

\begin{tabular}{cccc}
$\omega / H z$ & $A / m m$ & $K_{1} / K N . m^{-1}$ & $K_{3} / K N . m^{-3}$ \\
\hline 2 & 0.3 & 2.74 & 4.62 \\
6 & 0.3 & 2.45 & 4.80 \\
8 & 0.3 & 2.37 & 4.87 \\
10 & 0.3 & 2.33 & 4.95 \\
2 & 0.5 & 1.74 & 5.06 \\
6 & 0.5 & 1.44 & 5.22 \\
8 & 0.5 & 1.41 & 5.26 \\
10 & 0.5 & 1.35 & 5.32 \\
2 & 0.7 & 0.92 & 4.46 \\
6 & 0.7 & 0.80 & 4.56 \\
8 & 0.7 & 0.78 & 4.59 \\
10 & 0.7 & 0.73 & 4.60 \\
\hline
\end{tabular}

\subsection{Frequency and amplitude dependence}

It could be seen from Table 2 that the first order dynamic stiffness decreases slightly along with the rising frequency across the range of all investigated displacement amplitudes, whereas the third order stiffness increases. Besides, the first order dynamic stiffness decreases along with the increasing displacement amplitude, while the third order dynamic stiffness becomes larger before displacement amplitude increasing to $0.5 \mathrm{~mm}$ and after that decreases until amplitude increasing to $0.7 \mathrm{~mm}$. In order to capture this frequency-dependent and amplitude-dependent characteristics of the first and the third order dynamic stiffness, the identified results in Table 2 are further studied through mathematical interpolation and simulation. 
By relying on numerical analysis software, the first order dynamic stiffness is simulated as a function of displacement amplitude in the form of

$$
K_{1}(\omega, A)=a_{0}(\omega)+a_{1}(\omega) \cdot A+a_{2}(\omega) \cdot A^{2}
$$

All identified parameters of the first order dynamic stiffness at each excitation frequency $a_{i(i=0,1,2)}$ are summarized in Table 3.

\section{Table 3}

Identified parameters of the first order dynamic stiffness

\begin{tabular}{cccc}
\hline $\begin{array}{c}\text { Frequency } \\
\omega / \mathrm{Hz}\end{array}$ & $a_{0}$ & $a_{1}$ & $a_{2}$ \\
\hline 2 & 4.594 & -6.854 & 2.292 \\
6 & 4.677 & -8.815 & 4.687 \\
8 & 4.471 & -8.270 & 4.289 \\
10 & 4.457 & -8.438 & 4.448 \\
\hline
\end{tabular}

It is worth noting that the parameters of the first order dynamic stiffness $a_{i(i=0,1,2)}$ are closely associated with frequency $\omega$ as Table 3 shown, which means that it is reasonable to express $a_{i(i=0,1,2)}$ as the function of frequency in equation (7).

Before presenting the expression of $a_{i(i=0,1,2)}$ varying as a function of frequency, the identified data as well as comparison results between interpolated and simulated coefficients $a_{i(i=0,1,2)}$ are demonstrated in Fig.6. From Fig.6, equation $y=A+B^{*} x+C^{*} x^{\wedge} 2+D^{*} x^{\wedge} 3$ with identified parameters $A, B, C$ and $D$ in Fig.6-1, Fig.6-2 and Fig.6-3 can simulate the relationship between parameters $a_{i(i=0,1,2)}$ and frequency with good accuracy. The relation between all the coefficients $a_{i(i=0,1,2,3)}$ and frequency could be written as a matrix equation in the form of

$$
\left(\begin{array}{lll}
a_{0} & a_{1} & a_{2}
\end{array}\right)=\left(\begin{array}{llll}
1 & \omega & \omega^{2} & \omega^{3}
\end{array}\right) \cdot b_{K_{1}},
$$

where

$$
b_{k_{1}}=\left(\begin{array}{ccc}
3.77 & -1.75 & -2.93 \\
0.61 & -3.56 & -3.59 \\
-0.11 & 0.56 & -0.54 \\
0.01 & -0.03 & 0.03
\end{array}\right)
$$

Inserting equation (8) into (7), the first order dynamic stiffness is obtained as 


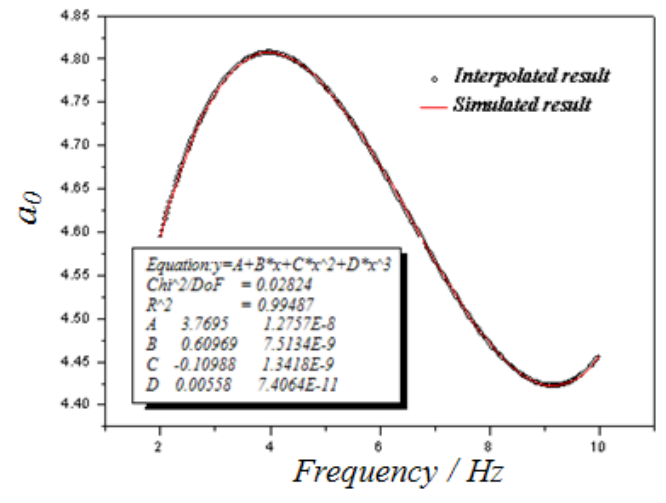

Fig.6-1 Identified results of coefficient $a_{0}$

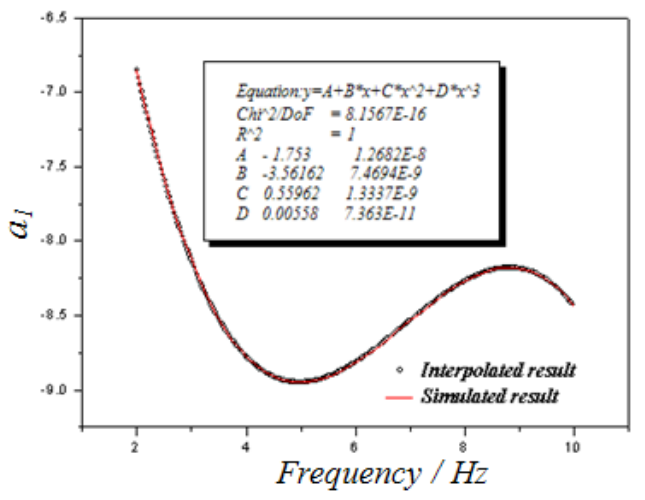

Fig.6-2 Identified results of coefficient $a_{1}$

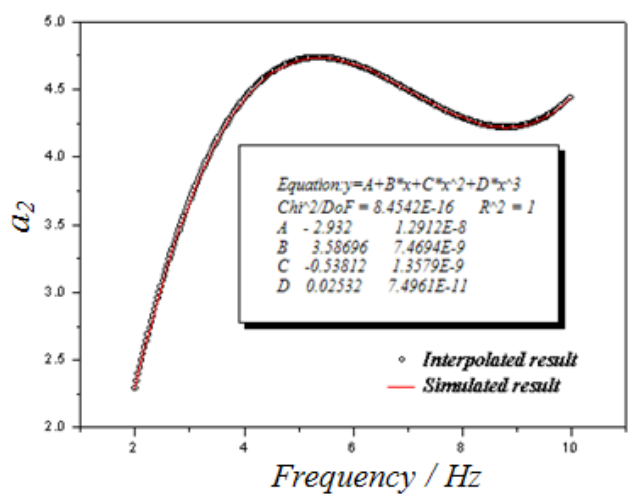

Fig.6-3 Identified results of coefficient $a_{2}$

Fig.6 Identified data and comparison results between interpolated and simulated coefficients $a_{i(i=0,1,2)}$ in equation (7)

$$
K_{1}(\omega, A)=\left(\begin{array}{lll}
a_{0} & a_{1} & a_{2}
\end{array}\right) \cdot\left(\begin{array}{lll}
1 & A & A^{2}
\end{array}\right)^{T}=\left(\begin{array}{llll}
1 & \omega & \omega^{2} & \omega^{3}
\end{array}\right) \cdot b_{K_{1}} \cdot\left(\begin{array}{lll}
1 & A & A^{2}
\end{array}\right)^{T} .
$$

Similarly, the third order dynamic stiffness could therefore be given by the same method as

$$
K_{3}(\omega, A)=\left(\begin{array}{lll}
a_{0} & a_{1} & a_{2}
\end{array}\right) \cdot\left(\begin{array}{lll}
1 & A & A^{2}
\end{array}\right)^{T}=\left(\begin{array}{llll}
1 & \omega & \omega^{2} & \omega^{3}
\end{array}\right) \cdot b_{K_{3}} \cdot\left(\begin{array}{lll}
1 & A & A^{2}
\end{array}\right)^{T},
$$

where

$$
b_{k_{3}}=\left(\begin{array}{ccc}
2.11 & 11.28 & -11.43 \\
-0.08 & 0.87 & -1.03 \\
0.02 & -0.15 & 0.07 \\
0 & 0.01 & -0.01
\end{array}\right) \text {. }
$$

In terms of equation (6), the model of restoring force of rubber absorber in rail fastening exposed to harmonic excitations finally is

$$
F_{\text {Restoring }}=\Omega \cdot b_{K_{1}} \cdot \Lambda^{\mathrm{T}} \cdot x+\Omega \cdot b_{K_{3}} \cdot \Lambda^{\mathrm{T}} \cdot x^{3} \pm \frac{E}{\pi A} \sqrt{1-\frac{\left(x-x_{0}\right)^{2}}{A^{2}}},
$$


where $\Omega=\left(1 \quad \omega \quad \omega^{2} \quad \omega^{3}\right), \Lambda=\left(1 \quad A \quad A^{2}\right), \omega$ is frequency $, H z, A$ is amplitude, $m m$.

\section{Results and discussion}

The hysteresis loop of rubber absorber in rail fastening at a single sinusoidal displacement excitation, whose frequency is $6 \mathrm{~Hz}$ and displacement amplitude is $0.3 \mathrm{~mm}$ (i.e. one of the excitation case above), is shown in Fig.7. The simulated results depicted as red rhombus are compared with those from measurement shown as black squares as is shown in this figure. The presented good agreement between simulated and measured results inevitably leads to a conclusion that the proposed nonlinear model in this investigation is capable of simulating the hysteresis loop of rubber absorber in rail fastening at its own special working conditions with good accuracy. This result confirms that rubber absorber in rail fastening exhibits strong nonlinear properties, which are mainly expressed by the nonlinear damping and the dynamic nonlinear stiffness. It is important to note that the single excitation case in Fig.7 is just taken as an example, and the simulated results of all the other cases in fact show good agreements as well with the meaured counterparts.

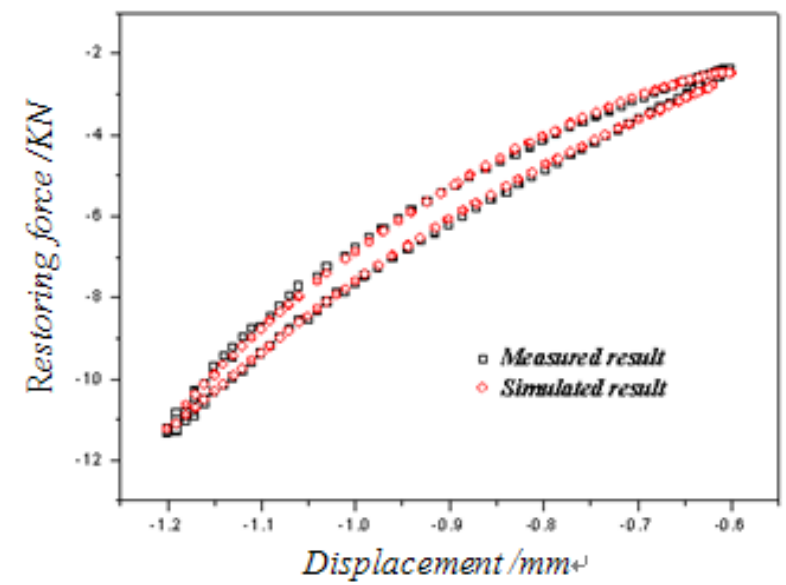

Fig.7 Comparison between force-displacement loops from measurement and simulation using the model shown in equation (13) at a single excitation case as Frequency is $6 \mathrm{~Hz}$ and amplitude is $0.3 \mathrm{~mm}$

In order to make out the amplitude dependency and frequency dependency, Fig.8 presents the relationship between dynamic nonlinear stiffness, excitation frequency and displacement amplitude in the form of three-dimensional surface using the verified expressions (10) and (11) based on MATLAB platform, which enables us to observe visually and conveniently.

As is shown in the Fig.8, frequency dependences of the first order dynamic stiffness as well as the third order dynamic stiffness are not as great as amplitude dependency. An 
increase in amplitude would decrease the first order dynamic stiffness largely as shown in Fig.8-1, whereas from the right graph an increase in frequency diminishes the dynamic stiffness very slightly as the displacement amplitude is $0.3 \mathrm{~mm}$, and this downward trend is becoming very hard to recognize as the amplitude increasing to $0.7 \mathrm{~mm}$. Varying the frequency from 2 to $10 \mathrm{~Hz}$ results in a slight increment of the third order dynamic stiffness from Fig.8-2. However, an increase in amplitude from 0.3 to approximate $0.5 \mathrm{~mm}$ leads to an increment of the third order dynamic stiffness across the range of all investigated frequencies, and, after that, the third order dynamic stiffness decreases rapidly along with the varying amplitude. This complex trend of the third order dynamic stiffness with displacement amplitude probably follows the Payne effect [23], which can to some degree explain the upward trend at the very beginning. Further, the discussion about
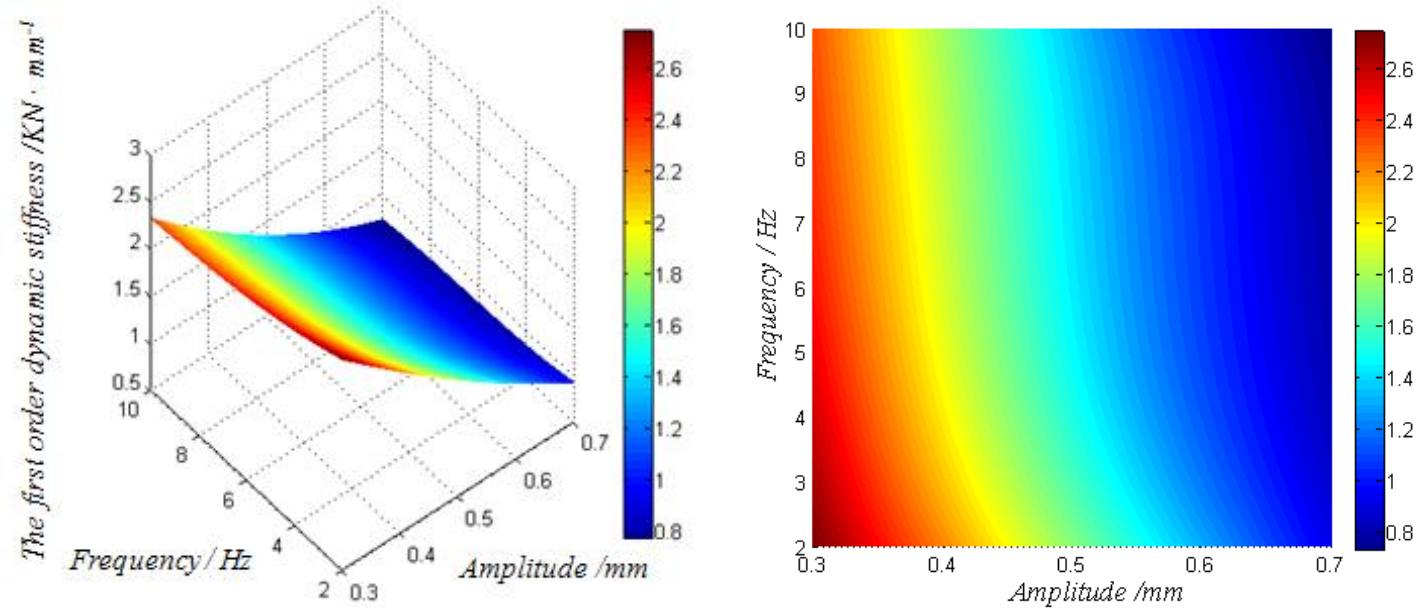

Fig.8-1 The first order dynamic stiffness
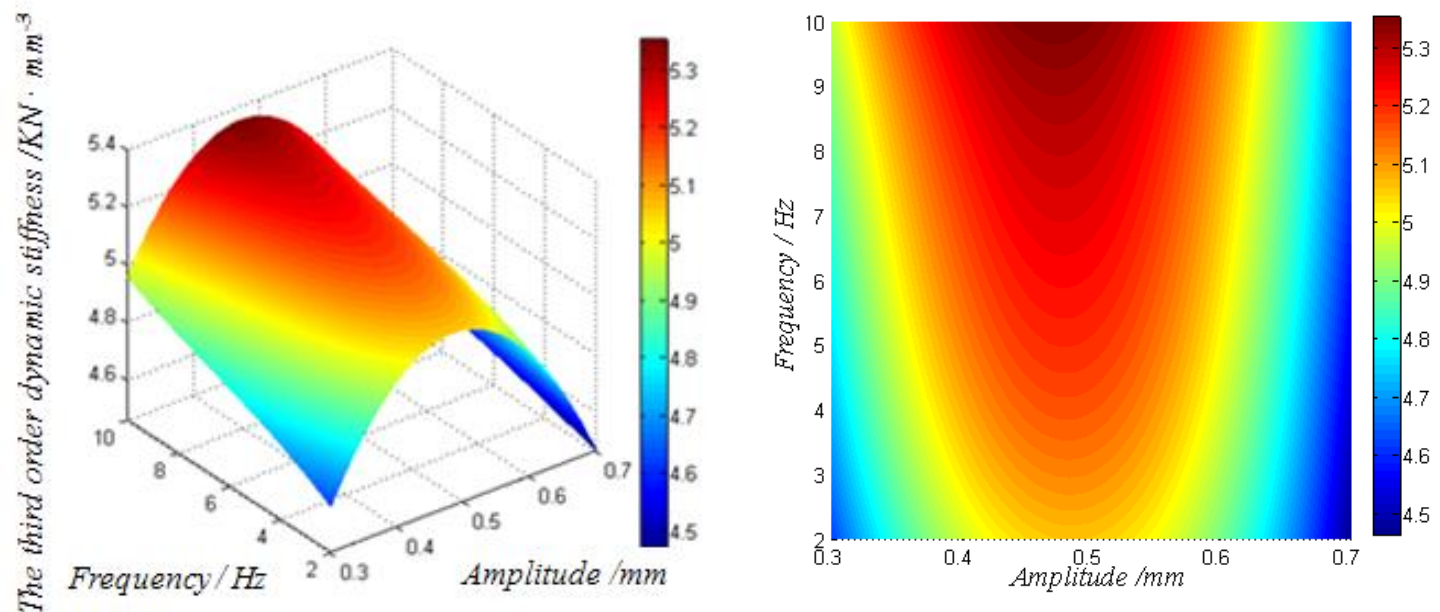

Fig.8-2 The third order dynamic stiffness

Fig.8 Frequency and amplitude dependency of dynamic nonlinear stiffness 
amplitude and frequency dependency here is based on the verified dynamic nonlinear model shown in equations (10) and (11), which is generally in accordance with Table 2. As for the reason why there are so many differences between the first and the third order dynamic stiffness at their amplitude as well as frequency dependency, it needs further investigation.

\section{Conclusion}

Rubber absorber in rail fastening exposed to single harmonic excitation with an initial displacement is found to exhibit a strong nonlinear behavior through a dynamic nonlinear model proposed in this paper. The key strategy of this method is to simulate the hysteresis loop of rubber components based on the superposition principle, which means that the restoring force of rubber absorber consisting of a nonlinear damping force and a nonlinear elastic force. In this model, the dynamic nonlinear stiffness was also defined. In order to determine model parameters and verify the accuracy of proposed model, a dynamic experiment is designed. The obtained simulated results show good agreement with the measured counterparts. This conclusion indicates that the proposed dynamic nonlinear model is therefore verified to be an effective mechanical tool in simulation and characterization of dynamic nonlinear behavior of rubber absorber in rail fastening with certain modes of vibrations. Besides, excitation frequency dependency as well as displacement amplitude dependency of the dynamic nonlinear stiffness were further studied and discussed. The result shows that the characteristic of dynamic stiffness is closely associated with both displacement amplitude and frequency. An increase in frequency diminishes the first order dynamic stiffness very slightly but leads to a small increment on the third order dynamic stiffness. Varying the amplitude upwards decreases the first order dynamic stiffness but results in a much more complex influence on the third order dynamic stiffness --- increasing it at first and then decreasing it rapidly. Generally, the frequency dependency of dynamic stiffness is not as great as the amplitude dependency.

\section{Reference}

[1] J. Harris, A. Stevenson, On the role of nonlinearity in the dynamic behavior of rubber components, Rubber Chem. Tech 59 (5) (1986) 740-764.

[2] A.I. Medalia, G. Kraus, Reinforcement of elastomers by particulate fillers, Science and Technology of Rubber, Academic Press, San Diego, (1944), 387-418.

[3] A.R. Payne, R.E. Whittaker, Low strain dynamic properties of filled rubbers, 
Rubber Chem. Tech 44 (1971) 440-478.

[4] A.I. Medalia, Effects of carbon black on dynamic properties of rubber, Rubber Chem. Tech 51 (1978) 437-523.

[5] M.J. Gregory, Dynamic properties of rubber in automotive engineering, Elastomerics 117 (11) (1985) 19-24.

[6] L. Mullins, Softening of rubber by deformation, Rubber Chem. Tech 42 (1969) 339-362.

[7] L. Mullins, Mechanical behavior of polyemers --- Accomplishments and problems, C. Hepburn, R.J.W. Reynods, Criteria for engineering design, Applied Science Publishers, (1979) 1-18.

[8] G.D. Dean, J.C. Duncan, A.F. Johnson, Determination of non-linear dynamic properties of carbon-filled rubbers, Polym. Tst. 4(2-4) (1984) 225-249.

[9] M. Sjöberg, Dynamic behavior of a rubber component in the low frequency range, Measurements and modeling, Proceedings of the $7^{\text {th }}$ International Conference of Sound and Vibration, Garmisch-Partenkirchen, (2000), 2955-2962.

[10] J.G. Sommer, D.A. Meyer, Factors controlling the dynamic properties of elastomeric products. SAE Paper No 730267, 1973.

[11] M.J. Wang, Effect of polymer-filler and filler-filler interactions on dynamic properties of filled vulcanizates. Rubber Chem. Tech 71 (3) (1998) 520-589.

[12] M.J. Wang, W.J. Pattersson, G.B. Ouyang, Dynamic stress-softening of filled vulcanizates. Kautsch Gummi Kunstst 51 (2) (1998) 106-121.

[13] J.A. Harris, Dynamic testing under nonsinusiodal conditions and the consequencs of nonlinearity for service performance, Rubbr Chem. Tech 60 (5) (1987) 870-887.

[14] D.M. Turner, D.Boast, M. Marfell, Changes in the behavior of rubber components as a consequence of real road conditions, C577/019/2000, ImechE Conference Transactions-Vehicle Noise and Vibration, (2000) 33-41.

[15] V.A. Coveney, D.E. Johnson, D.M. Turner, A triboelastic model for the cyclic mechanical behavior of filled vulcanizates, Rubber Chem. Tech 68 (1985) 660-670.

[16] P. Zeng, G.P. Yan, Influence of dynamic characteristics of lead-rubber bearing on seismic response of continuous beam bridge, Journal of railway society 23 (5) (2011) 96-101.

[17] M.X. Fang, Q.C. Feng, H. Jiang, Simulating Research of Automobile Hysteresis Nonlinear Dynamic System, Automobile Technology, (11) (2006). 
[18] I.G. Buckle, R.L. Mayes, Seismic Isolation, History application and performance, A world view, Earthquake spectra, 2 (6) (1990).

[19] Xiao X B , Jin X S, Wen Z F, Effect of disabled fastening systems and ballast on vehicle derailment, Vib. Acoust 129 (2) (2007) 217-230.

[20] Bezin Y, Iwnicki SD, An investigation of sleeper voids using a flexible track model integrated with railway muti-body dynamics, Proceedings of the institution of mechanical engineers part, F-journal of rail and rapdi transit, 223 (6) (2009) 597-607.

[21] S. Singiresu, Mechanical vibrations, 2009.

[22] R.M. Christensen, Theory of viscoelasticity, 11 (1990).

[23] M. Sjöberg, Kari L Testing of nonlinear interaction effects of sinusoidal and noise excitation on rubber isolator stiffness, Polymer Testing, (22) (2003) 343-351. 